\title{
Homotopical aspects of commutative algebras I: freeness conditions for crossed squares
}

\author{
Zekeriya Arvasi · Erdal Ulualan
}

Received: 23 August 2012 / Accepted: 30 November 2013 / Published online: 13 December 2013

(C) Tbilisi Centre for Mathematical Sciences 2013

\begin{abstract}
We give an alternative description of the top algebra of the free crossed square of algebras on 2-construction data in terms of tensors and coproducts of crossed modules of commutative algebras.
\end{abstract}

Keywords Free crossed modules - Simplicial algebras · Crossed squares · Crossed complexes

Mathematics Subject Classification (2010) $\quad$ 18G30 - 18G35 · 18G55

\section{Introduction}

Ellis [12] gave a description of the free crossed square of groups of a CW-complex using topological methods. In contexts other than groups, he has defined crossed squares, 2-crossed modules etc. in [12]. For commutative algebras, 2-crossed modules have been defined by Grandjean and Vale [13].

Combining earlier work [17] of Porter with Arvasi and Porter's joint papers [5-7], one starts to see how a study of the links between simplicial commutative algebras and classical constructions of homological algebra can be strengthened by interposing

Communicated by Graham Ellis.

Z. Arvasi

Department of Mathematics-Computer, Science and Art Faculty,

Eskişehir Osmangazi University, 26480 Eskisehir, Turkey

e-mail: zarvasi@ogu.edu.tr

E. Ulualan $(\bowtie)$

Department of Mathematics, Science and Art Faculty, Dumlupınar University, Kutahya, Turkey

e-mail: erdal.ulualan@dpu.edu.tr; eulualan@gmail.com 
crossed algebraic models for the homotopy types of simplicial algebras. In this note, we continue this process using these methods to give a description in terms of tensor products, of the top corner of a free crossed square of commutative algebras and the top term of the corresponding free 2 -crossed module. The methods are slightly different, but these results are 2-dimensional analogues of the description of the free crossed module on a 'presentation' of an algebra in terms of Koszul-like terms, given in [17].

We end with a section looking at possible links of this with André-Quillen homology and a squared complex form of the cotangent complex (cf. [15]).

The results and general methods we use are inspired by those given for the corresponding case of groups in [16]. Some of the methods of that paper, of course, go across almost verbatim to this commutative algebra case, but, as the audience for this paper is probably more or less disjoint from that for [16] it seems advisable to repeat arguments from that paper in this algebra case even when they might be safely 'left to the reader'. Of course, there are all times when the translation between the two contexts is less easy.

\section{Preliminaries}

Let $\mathbf{k}$ be a fixed commutative ring with identity. By a k-algebra, we mean a unitary k-bimodule $C$ endowed with a k-bilinear associative multiplication $C \times C \rightarrow C$, $\left(c, c^{\prime}\right) \mapsto c c^{\prime}$. The algebra $C$ will as usual be called commutative if $c c^{\prime}=c^{\prime} c$ for all $c, c^{\prime} \in C$. In this work, all algebras will be commutative and will be over the same fixed commutative ring $\mathbf{k}$. We will denote the category of all algebras by Alg.

\subsection{Simplicial algebras}

Denoting the usual category of finite ordinals by $\Delta$, we obtain for each $k \geq 0$ a subcategory $\Delta_{\leq k}$ determined by the objects [j] of $\Delta$ with $j \leq k$. A simplicial algebra is a functor from the opposite category $\Delta^{o p}$ to Alg; a $k$-truncated simplicial algebra is a functor from $\left(\Delta_{\leq k}^{o p}\right)$ to Alg. We denote the category of simplicial algebras by SimpAlg and the category of $k$-truncated simplicial algebras by $\operatorname{Tr}_{\mathbf{k}} \operatorname{SimpAlg}$. By a $k$-truncation of a simplicial algebra, we mean a $k$-truncated simplicial algebra $\operatorname{tr}_{k} \mathbf{E}$ obtained by forgetting dimensions of order $>k$ in a simplicial algebra $\mathbf{E}$, that is restricting $\mathbf{E}$ to $\Delta_{\leq k}^{o p}$. This gives a truncation functor

$$
\operatorname{tr}_{k}: \operatorname{SimpAlg} \longrightarrow \operatorname{Tr}_{k} \operatorname{SimpAIg}
$$

which admits a right adjoint

$$
\operatorname{cosk}_{k}: \operatorname{Tr}_{k} \operatorname{SimpAlg} \longrightarrow \operatorname{SimpAlg}
$$

called the $k$-coskeleton functor, and a left adjoint

$$
\mathbf{s k}_{\mathbf{k}}: \operatorname{Tr}_{\mathbf{k}} \text { SimpAlg } \longrightarrow \text { SimpAlg }
$$


called the $k$-skeleton functor. For explicit construction of these see [9]. We will say that a simplicial algebra $\mathbf{E}$ is $k$-skeletal if the natural morphism $\left(\mathbf{s k}_{k} \circ \mathbf{t r}_{k}\right)(\mathbf{E}) \rightarrow \mathbf{E}$ is an isomorphism.

Recall that given a simplicial algebra $\mathbf{E}$, the Moore complex (NE, $\partial$ ) of $\mathbf{E}$ is the chain complex defined by

$$
(N E)_{n}=\bigcap_{i=0}^{n-1} \operatorname{Ker} d_{i}^{n}
$$

with $\partial_{n}: N E_{n} \rightarrow N E_{n-1}$ induced from $d_{n}^{n}$ by restriction.

The $n$th homotopy module $\pi_{n}(\mathbf{E})$ of $\mathbf{E}$ is the nth homology of the Moore complex of $\mathbf{E}$, i.e.,

$$
\begin{aligned}
\pi_{n}(\mathbf{E}) & \cong H_{n}(\mathbf{N E}, \partial) \\
& =\bigcap_{i=0}^{n} \operatorname{Ker} d_{i}^{n} / d_{n+1}^{n+1}\left(\bigcap_{i=0}^{n} \operatorname{Ker} d_{i}^{n+1}\right) .
\end{aligned}
$$

We say that the Moore complex NE of a simplicial algebra is of length $k$ if $N E_{n}=0$ for all $n \geq k+1$ so that a Moore complex is of length $k$ also of length $r$ for $r \geq k$. For example, if $\mathbf{E}$ has Moore complex of length 1 , then $\left(N E_{1}, N E_{0}, \partial_{1}\right)$ is a crossed module and conversely. If $\mathbf{N E}$ is of length 2, the corresponding Moore complex gives a 2-crossed module (cf. [7]).

\subsection{Free simplicial algebras}

Recall from [5] how to define a free simplicial algebra by using the 'step-by-step' construction of André [1].

Let $[n]$ be the ordered set, $[n]=\{0<1<\cdots<n\}$. There is an increasing surjective map $\alpha_{i}^{n}$ from $[n+1]$ to $[n]$ given by $\alpha_{i}^{n}(x)=x$ for $x \leqslant i$ and $\alpha_{i}^{n}(x)=x-1$ for $x>i$. We denote by $\{m, n\}$ the set of increasing surjective maps from $[m]$ to $[n]$ as used in [5].

Let $\mathbf{E}$ be a simplicial algebra and let $k \geq 1$ be fixed. Suppose we are given a set $\Omega$ of elements $\left\{x_{\lambda} \in \pi_{k-1}(\mathbf{E}): \lambda \in \Lambda\right\}$, then we can choose a corresponding set of elements $\omega_{\lambda} \in N E_{k-1}$ so that $x_{\lambda}=\omega_{\lambda}+\partial_{k}\left(N E_{k}\right)$. We want to define a simplicial algebra, $\mathbf{F}=\mathbf{E}[\Omega]$ with a monomorphism $i: \mathbf{E} \rightarrow \mathbf{F}$ such that $\pi_{k-1}(i): \pi_{k-1}(\mathbf{E}) \rightarrow \pi_{k-1}(\mathbf{F})$ 'kills off' the $x_{\lambda}$ 's. We do this by adding new indeterminates into $N E_{k}$ to enlarge it so as to make $i\left(\omega_{\lambda}\right) \in \partial\left(N F_{k}\right)$. More precisely,

1. $F_{n}=E_{n}\left[y_{\lambda, t}\right]$ with $\lambda \in \Lambda$ and $t$ is an element of the set $\{n, k\}$,

2. For $0 \leqslant i \leqslant n$, the homomorphisms $s_{i}^{n}: F_{n} \rightarrow F_{n+1}$ are obtained from the homomorphisms $s_{i}^{n}: E_{n} \rightarrow E_{n+1}$ with the relations $s_{i}^{n}\left(y_{\lambda, t}\right)=y_{\lambda, u}$ with $u=$ $t \alpha_{i}^{n}, t:[n] \rightarrow[k]$,

3. For $0 \leqslant i \leqslant n \neq 0$, the homomorphisms $d_{i}^{n}: F_{n} \rightarrow F_{n-1}$ are obtained from the homomorphisms $d_{i}^{n}: E_{n} \rightarrow E_{n-1}$ with the relations given in [5]. 
Thus we can give briefly the definition of a free simplicial algebra as follows. A simplicial algebra $\mathbf{F}$ is called a free if

i. $F_{n}=E_{n}$ for $n<k$,

ii. $F_{k}=$ a free $E_{k}$-algebra over a set of non-degenerate indeterminates, all of whose faces are zero except the $k$ th,

iii. $F_{n}$ is a free $E_{n}$-algebra over the degenerate elements for $n>k$.

Remark if $\mathbf{A}$ is a simplicial algebra, then there exists a free simplicial algebra $\mathbf{E}$ and an epimorphism $\mathbf{E} \rightarrow \mathbf{A}$ which induces isomorphisms on all homotopy modules. The details are omitted as they are 'well-known'.

\subsection{Crossed modules of algebras}

We recall that if $C$ and $R$ are commutative k-algebras, a map $R \times C \rightarrow C,(r, c) \mapsto r \cdot c$, is a commutative action if and only if

1. $k(r \cdot c)=(k r) \cdot c=r \cdot(k c)$

2. $r \cdot\left(c+c^{\prime}\right)=r \cdot c+r \cdot c^{\prime}$

3. $\left(r+r^{\prime}\right) \cdot c=r \cdot c+r^{\prime} \cdot c$

4. $r \cdot\left(c c^{\prime}\right)=(r \cdot c) c^{\prime}=c\left(r \cdot c^{\prime}\right)$

5. $\left(r r^{\prime}\right) \cdot c=r \cdot\left(r^{\prime} \cdot c\right)$

for all $k \in \mathbf{k}, c, c^{\prime} \in C$ and $r, r^{\prime} \in R$.

If $C$ and $R$ are commutative $\mathbf{k}$-algebras and $R$ has a commutative action on $C$, then $C$ is also an $R$-algebra. Throughout this paper we denote an action of $r \in R$ on $c \in C$ by $r \cdot c$ and we denote the multiplication of $c, c^{\prime}$ in $C$ by $c c^{\prime}$.

Crossed modules of groups were initially defined by Whitehead in [20]. The commutative algebra analogue has been studied by Porter in [17].

Let $R$ be a k-algebra with identity. A pre-crossed module of commutative algebras is an $R$-algebra $C$ together with a commutative action of $R$ on $C$ and an algebra morphism $\partial: C \rightarrow R$ such that for all $c \in C, r \in R$,

$$
\text { 1. } \partial(r \cdot c)=r \partial c \text {. }
$$

This is a crossed module if in addition, for all $c, c^{\prime} \in C$ and

$$
\text { 2. } \partial(c) \cdot c^{\prime}=c c^{\prime} \text {. }
$$

The second condition is called the Peiffer identity.

Examples of crossed modules are:

i. Any ideal, $I$, in $R$ gives an inclusion map $I \longrightarrow R$, which is a crossed module. Conversely, given any crossed $R$-module $\mu: C \longrightarrow R$, the image $I=\mu(C)$ of $C$ is an ideal in $R$.

ii. Any $R$-module $M$ can be considered as an $R$-algebra with zero multiplication and hence $M \stackrel{0}{\longrightarrow} R$ is a crossed $R$-module. Conversely, if $\mu: C \longrightarrow R$ is a crossed $R$-module, $\operatorname{Ker} \mu$ is an $R / \mu(C)$-module. 


\section{Crossed squares and simplicial algebras}

Although we will be mainly concerned with crossed squares in this paper, some of the arguments either clearly apply or would seem to apply in the more general case of crossed $n$-cubes and $n$-cube complexes.

Crossed $n$-cubes in algebraic settings such as commutative algebras, Jordan algebras, Lie algebras have been defined by Ellis [10,11].

A crossed $n$-cube of commutative algebras is a family of commutative algebras, $M_{A}$ for $A \subseteq\langle n\rangle=\{1, \ldots, n\}$ together with homomorphisms $\mu_{i}: M_{A} \rightarrow M_{A-\{i\}}$ for $i \in\langle n\rangle$ and for $A, B \subseteq\langle n\rangle$, functions

$$
h: M_{A} \times M_{B} \longrightarrow M_{A \cup B}
$$

such that for all $k \in \mathbf{k}, a, a^{\prime} \in M_{A}, b, b^{\prime} \in M_{B}, c \in M_{C}, i, j \in\langle n\rangle$ and $A \subseteq B$

$$
\begin{array}{lc}
\text { 1. } & \mu_{i} a=a \quad \text { if } i \notin A \\
\text { 2. } & \mu_{i} \mu_{j} a=\mu_{j} \mu_{i} a \\
\text { 3. } & \mu_{i} h(a, b)=h\left(\mu_{i} a, \mu_{i} b\right) \\
\text { 4. } & h(a, b)=h\left(\mu_{i} a, b\right)=h\left(a, \mu_{i} b\right) \text { if } i \in A \cap B \\
\text { 5. } & h\left(a, a^{\prime}\right)=a a^{\prime} \\
\text { 6. } & h(a, b)=h(b, a) \\
\text { 7. } & h\left(a+a^{\prime}, b\right)=h(a, b)+h\left(a^{\prime}, b\right) \\
\text { 8. } & h\left(a, b+b^{\prime}\right)=h(a, b)+h\left(a, b^{\prime}\right) \\
\text { 9. } & k \cdot h(a, b)=h(k \cdot a, b)=h(a, k \cdot b) \\
\text { 10. } & h(h(a, b), c)=h(a, h(b, c))=h(b, h(b, c)) .
\end{array}
$$

A morphism of crossed n-cubes is defined in the obvious way: It is a family of commutative algebra homomorphisms, for $A \subseteq\langle n\rangle, f_{A}: M_{A} \longrightarrow M_{A}^{\prime}$ commuting with the $\mu_{i}^{\prime}$ s and $h^{\prime} s$. We thus obtain a category of crossed $n$-cubes denoted by $\mathrm{Crs}^{\mathrm{n}}$.

Example (1) For $n=1$, a crossed 1-cube is the same as a crossed module.

For $n=2$ one has a crossed square (cf. [14]):

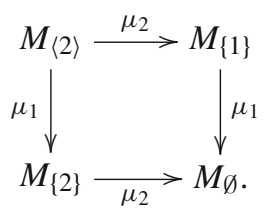

Each $\mu_{i}$ is a crossed module as is $\mu_{1} \mu_{2}$. The $h$-functions $h: M_{\emptyset} \times M_{\{1\}} \rightarrow M_{\{1\}}$ and $h: M_{\emptyset} \times M_{\{2\}} \rightarrow M_{\{2\}}$ give the actions of $M_{\emptyset}$ on $M_{\{1\}}$ and $M_{\{2\}}$, respectively. For example the action of $r \in M_{\emptyset}$ on $c \in M_{\{1\}}$ is given by $r \cdot c=h(r, c)$. Then $\mu_{1}$ is a crossed module since $\mu_{1}(c) \cdot c^{\prime}=h\left(\mu_{1} c, c^{\prime}\right)=h\left(c, c^{\prime}\right)=c c^{\prime}$. Also the $h$-function 


$$
h: M_{\{1\}} \times M_{\{2\}} \longrightarrow M_{\langle 2\rangle} .
$$

gives the $h$-map of the crossed square.

The maps $\mu_{2}$ (or $\left.\mu_{1}\right)$ also define a map of crossed modules from $\left(M_{\langle 2\rangle}, M_{\{2\}}, \mu_{1}\right)$ to $\left(M_{\langle 2\rangle}, M_{\emptyset}, \mu_{1}\right)$. In fact a crossed square can be thought of as a crossed module in the category of crossed modules.

(2) Let $I_{1}$ and $I_{2}$ be ideals of an algebra $E$. The commutative square diagram of inclusions;

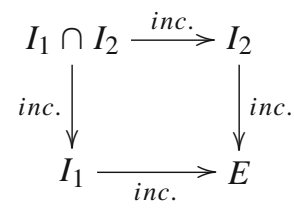

naturally comes together with actions of $E$ on $I_{1}, I_{2}$ and $I_{1} \cap I_{2}$ given by multiplication, and functions

$$
\begin{aligned}
h: I_{A} \times I_{B} & \longrightarrow I_{A} \cap I_{B}=I_{A \cup B} \\
(a, b) & \longmapsto a b .
\end{aligned}
$$

That this is a crossed square is easily checked.

(3) Let $\mathbf{E}$ be a simplicial algebra. Let $M(\mathbf{E}, 2)$ denote the following diagram

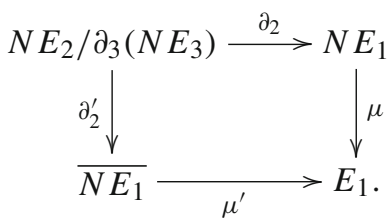

Then this is the underlying square of a crossed square. The extra structure is given as follows: $N E_{1}=\operatorname{Ker} d_{0}^{1}$ and $\overline{N E}_{1}=\operatorname{Ker} d_{1}^{1}$. Since $E_{1}$ acts on $N E_{2} / \partial_{3} N E_{3}, \overline{N E}_{1}$ and $N E_{1}$, there are actions of $\overline{N E}_{1}$ on $N E_{2} / \partial_{3} N E_{3}$ and $N E_{1}$ via $\mu^{\prime}$, and $N E_{1}$ acts on $N E_{2} / \partial_{3} N E_{3}$ and $\overline{N E}_{1}$ via $\mu$. As $\mu$ and $\mu^{\prime}$ are inclusions, all actions can be given by multiplication. The $h$-map is

$$
\begin{aligned}
N E_{1} \times \overline{N E}_{1} & \longrightarrow N E_{2} / \partial_{3} N E_{3} \\
(x, \bar{y}) & \longmapsto h(x, \bar{y})=s_{1} x\left(s_{1} y-s_{0} y\right)+\partial_{3} N E_{3},
\end{aligned}
$$

which is bilinear. Here $x$ and $y$ are in $N E_{1}$ as there is a natural bijection between $N E_{1}$ and $\overline{N E}_{1}$ (by [6, Lemma 2.1]). The element $\bar{y}$ is the image of $y$ under this. This example effectively introduces the functor

$$
\mathbf{M}(-, 2): \text { SimpAlg } \rightarrow \mathrm{Crs}^{2} .
$$


This is the case $n=2$ of a general construction of a crossed $n$-cube from a simplicial algebra given by the first author in [2] where the reader may find the verification of the axioms (this notational convention will be revisited at the end of Sect. 6).

Note if we consider the above crossed square as a vertical morphism of crossed modules, we can take its kernel and cokernel within the category of crossed modules. In the above the morphisms in the top left hand corner are induced from $d_{2}$ so

$$
\operatorname{Ker}\left(\partial_{2}^{\prime}: \frac{N E_{2}}{\partial_{3} N E_{3}} \longrightarrow \operatorname{Ker} d_{1}\right)=\frac{N E_{2} \cap \operatorname{Ker} d_{2}}{\partial_{3} N E_{3}} \cong \pi_{2}(\mathbf{E})
$$

whilst the other map labeled $\mu$ is an inclusion so has trivial kernel, hence the kernel of this morphism of crossed modules is

$$
\pi_{2}(\mathbf{E}) \longrightarrow 0
$$

The image of $\partial_{2}$ (and $\mu^{\prime}$ ) is an ideal in both the algebras on the bottom line and as $\operatorname{Ker} d_{0}=N E_{1}$ with the corresponding $\operatorname{Im} \mu$ being $d_{2} N E_{2}$, the cokernel is $N E_{1} / \partial_{2} N E_{2}$, whilst $E_{1} / \operatorname{Ker} d_{0} \cong E_{0}$, i.e, the cokernel of $\mu$ is $M(\mathbf{E}, 1)$.

In fact of course $\mu$ is not only a morphism of crossed modules, it is a crossed module. This means that $\pi_{2}(\mathbf{E}) \rightarrow 0$ is in some sense a $M(\mathbf{E}, 1)$-module, (cf. [3]), and that $M(\mathbf{E}, 2)$ can be thought of as a crossed extension of $M(\mathbf{E}, 1)$ by $\pi_{2}(\mathbf{E})$.

\section{Free crossed squares}

Ellis, [12], in 1993 presented the notion of a free crossed square for the case of groups. In this section, we introduce a commutative algebra version of this definition and give a construction of a free crossed square by using the second order Peiffer elements and the 2-skeleton of a 'step-by-step' construction of a free simplicial algebra.

We firstly adapt Ellis's definition of the free crossed square on a pair of functions $\left(f_{2}, f_{3}\right)$ to the algebra context:

Let $\mathbf{S}_{\mathbf{1}}, \mathbf{S}_{\mathbf{2}}$ and $\mathbf{S}_{\mathbf{3}}$ be sets which for simplicity we assume are finite. Suppose given a function $f_{2}: \mathbf{S}_{\mathbf{2}} \rightarrow R$ from a set $\mathbf{S}_{\mathbf{2}}$ to a free algebra $R$ on $\mathbf{S}_{\mathbf{1}}$. Let $\partial: M \rightarrow R$ be the free pre-crossed module on $f_{2}$. Using the action of $R$ on $M$ we can form the semi-direct product $M \rtimes R$. The inclusion $\mu: M \rightarrow M \rtimes R$ given by $m \mapsto(m, 0)$ enables us to take $M$ as an ideal of $M \rtimes R$. (Recall from examples of crossed modules that any ideal inclusion is a crossed module with action given by multiplication.) There is also another ideal of $M \rtimes R$ coming from $M$, namely

$$
\bar{M}=\{(m, r) \in M \rtimes R: \partial m=-r\}
$$

with inclusion denoted $\bar{\mu}: \bar{M} \rightarrow M \rtimes R$.

Assume given a function from a set $\mathbf{S}_{\mathbf{3}}$ to $M, f_{3}: \mathbf{S}_{\mathbf{3}} \rightarrow M$, which is to satisfy $\partial f_{3}=$ 0 . Then there is a corresponding function $\bar{f}_{3}: \mathbf{S}_{\mathbf{3}} \rightarrow \bar{M}$ given by $y \mapsto\left(f_{3}(y), 0\right)$. 
We say a crossed square

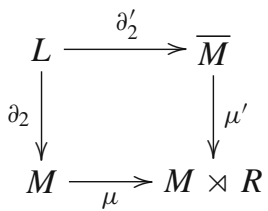

is totally free on the pair of functions $\left(f_{2}, f_{3}\right)$ if

i. $(M, R, \partial)$ is the free pre-crossed module on $f_{2}$;

ii. $\mathbf{S}_{3}$ is a subset of $L$ with $f_{3}$ and $\bar{f}_{3}$ the restrictions of $\partial_{2}$ and $\partial_{2}^{\prime}$ respectively;

iii. for any crossed square

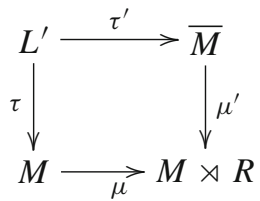

and any function $v: \mathbf{S}_{\mathbf{3}} \rightarrow L^{\prime}$ satisfying $\tau v=f_{3}$, there is a unique morphism $\Phi=(\phi, i d, i d, i d)$ of crossed squares:

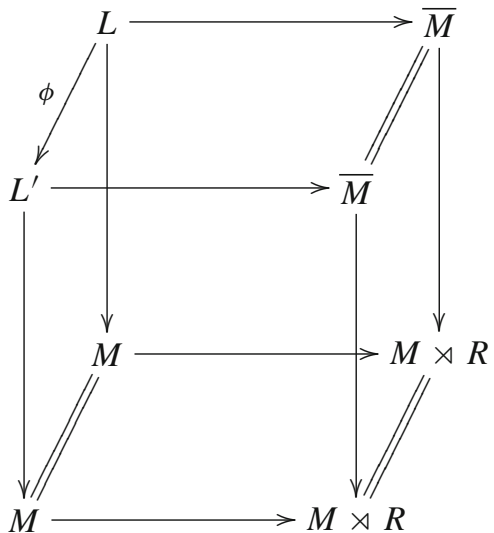

such that $\phi v^{\prime}=v$, where $v^{\prime}: \mathbf{S}_{\mathbf{3}} \rightarrow L$ is the inclusion.

We denote such a free crossed square of algebras by $(L, M, \bar{M}, M \rtimes R)$.

A free pre-crossed module on the function $f_{2}: \mathbf{S}_{2} \rightarrow R$ with codomain $R$ is given as follows. Consider $C=R^{+}\left[\mathbf{S}_{2}\right]$, the positively graded part of the polynomial ring on the set $\mathbf{S}_{2}$ with coefficients in $R$, so that $R$ acts on $C$ by multiplication. The function $f_{2}$ induces a morphism of $R$-algebras $\partial: R^{+}\left[\mathbf{S}_{2}\right] \rightarrow R$ given by $\partial(y)=f_{2}(y)$ and this is a free pre-crossed module on the function $f_{2}: \mathbf{S}_{2} \rightarrow R$. So, the function

$$
f_{3}: \mathbf{S}_{\mathbf{3}} \rightarrow M,\left(\text { with } M=R^{+}\left[\mathbf{S}_{\mathbf{2}}\right]\right)
$$


is precisely the data $\left(\mathbf{S}_{\mathbf{3}}, f_{3}\right)$ for 2-dimensional construction data in the simplicial context. We thus need to recall the 2-dimensional construction data in a free simplicial algebra (cf. [6]). This 2-dimensional form can be pictured by the diagram

$$
\mathbf{E}^{(2)}: \cdots\left(R\left[s_{0}\left(\mathbf{S}_{2}\right), s_{1} \mathbf{S}_{\mathbf{2}}\right]\right)\left[\mathbf{S}_{\mathbf{3}}\right] \underset{s_{0}, s_{1}}{\stackrel{d_{0}, d_{1}, d_{2}}{\rightleftarrows}} R\left[\mathbf{S}_{2}\right] \underset{s_{0}}{\stackrel{d_{0}, d_{1}}{\rightleftarrows}} R
$$

with the simplicial morphisms given as in [7]. Here $\mathbf{S}_{\mathbf{2}}=\left\{S_{1}, \ldots, S_{n}\right\}$ and $\mathbf{S}_{\mathbf{3}}=$ $\left\{S_{1}^{\prime}, \ldots, S_{m}^{\prime}\right\}$ are finite sets with $\pi_{0}\left(\mathbf{E}^{(2)}\right) \cong B=R /\left(t_{1}, \ldots, t_{n}\right)$ as an $R$-algebra where $t_{i}=\partial S_{i}$.

\subsection{Free crossed squares exist}

Theorem 4.1 A totally free crossed square $(L, M, \bar{M}, M \rtimes R)$ exists on the 2dimensional construction data and is given by $\mathbf{M}\left(\mathbf{E}^{(2)}, \mathbf{2}\right)$ where $\mathbf{E}^{(2)}$ is the 2-skeletal free simplicial algebra defined by the construction data.

Proof Suppose given the 2-dimensional construction data for a free simplicial algebra, $\mathbf{E}$, which we will take as above as the data for a totally free crossed square. We will not assume detailed knowledge of [5] so we start with $R$ and $f_{2}: \mathbf{S}_{\mathbf{2}} \rightarrow R$ and form $M=R^{+}\left[\mathbf{S}_{\mathbf{2}}\right]=\left(S_{1}, \ldots, S_{n}\right)$. This gives $\partial_{1}: R^{+}\left[\mathbf{S}_{\mathbf{2}}\right] \rightarrow R$ as free pre-crossed module on $f_{2}$. The semidirect product gives us back

$$
R\left[\mathbf{S}_{2}\right] \cong M \rtimes R
$$

and we can identify this with $E_{1}^{(2)}$. This identification also makes

$$
M \cong \operatorname{Ker} d_{0}^{1}
$$

for the $d_{0}^{1}$ of $\mathbf{E}^{(2)}$.

Next form $\bar{M}=\{(m, r) \in M \rtimes R: \partial m=-r\}$. As $m \in R^{+}\left[\mathbf{S}_{\mathbf{2}}\right]$, writing $m=\sum r_{\alpha} S^{\alpha}$ for multi-indices $\alpha$, we get $\partial m=\sum r_{\alpha} t^{\alpha}$ where $t_{i}=f_{2}\left(S_{i}\right)$. Thus we can identify $\bar{M}$ with $\left(S_{1}-t_{1}, \ldots, S_{n}-t_{n}\right)$ which is exactly $\operatorname{Ker} d_{1}^{1}$ (for this see [5]).

Now $f_{3}: \mathbf{S}_{\mathbf{3}} \rightarrow \operatorname{Ker}_{1}=\operatorname{Ker}\left(\partial: N E_{1}^{(2)} \rightarrow N E_{0}^{(2)}\right) \subset R^{+}\left[\mathbf{S}_{2}\right]$. We know that this allows us to construct $E_{2}^{(2)}$, and hence $E_{n}^{(2)}$ for $n \geq 3$, and in addition that taking

$$
L=N E_{2}^{(2)} / \partial_{3}\left(N E_{3}^{(2)}\right),
$$

gives a crossed square

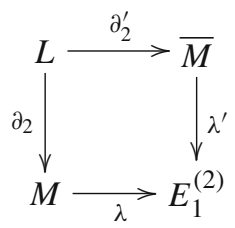


which is $M\left(\mathbf{E}^{(2)}, 2\right)$. We claim this is the totally free crossed square on the construction data.

At this stage it is worth nothing that there would seem to be no simple adjointness statement between $\mathbf{M}(-, 2)$ and some functor that would give a quick proof of freeness. The problem being that $\mathbf{M}(-, 2)$ seems to be an adjoint only up to some sort of coherent homotopy. To avoid this difficulty we use a more combinatorial approach involving the higher order Peiffer elements and a more concrete description of $L$.

In [5], the first author and Porter analyzed in general the structure of algebras of boundaries such as $\partial_{3}\left(N E_{3}^{(2)}\right)$. There they showed that $N E_{1}^{(2)}$ is generated as an ideal by elements of the following forms:

For all $x \in N E_{1}^{(2)}, y \in N E_{2}^{(2)}$,

$$
\begin{aligned}
& C_{(1,0)(2)}(x \otimes y)=\left(s_{1} s_{0} x-s_{2} s_{0} x\right) s_{2} y, \\
& C_{(2,0)(1)}(x \otimes y)=\left(s_{2} s_{0} x-s_{2} s_{1} x\right)\left(s_{1} y-s_{2} y\right), \\
& C_{(2,1)(0)}(x \otimes y)=s_{2} s_{1} x\left(s_{0} y-s_{1} y+s_{2} y\right) ;
\end{aligned}
$$

whilst for all $x, y \in N E_{2}$,

$$
\begin{aligned}
& C_{(1)(0)}(x \otimes y)=s_{1} x\left(s_{0} y-s_{1} y\right)+s_{2}(x y), \\
& C_{(2)(0)}(x \otimes y)=\left(s_{2} x\right)\left(s_{0} y\right), \\
& C_{(2)(1)}(x \otimes y)=s_{2} x\left(s_{1} y-s_{2} y\right) .
\end{aligned}
$$

We know that $\partial_{3}\left(N E_{3}^{(2)}\right)$ is generated by elements of the forms

$$
\left(s_{1} s_{0} d_{1} S_{i}-s_{0} S_{i}\right) S_{j}^{\prime}, \quad\left(s_{0} S_{i}-s_{1} S_{i}\right)\left(s_{1} d_{2} S_{j}^{\prime}-S_{j}^{\prime}\right), \quad s_{1} S_{i}\left(s_{0} d_{2} S_{j}^{\prime}-s_{1} d_{2} S_{j}^{\prime}+S_{j}^{\prime}\right),
$$

and for $S_{i}^{\prime}, S_{j}^{\prime} \in \mathbf{S}_{\mathbf{2}}$,

$S_{i}^{\prime}\left(s_{1} d_{2} S_{j}^{\prime}-S_{j}^{\prime}\right), S_{i}^{\prime}\left(S_{j}^{\prime}+s_{0} d_{2} S_{j}^{\prime}-s_{1} d_{2} S_{j}^{\prime}\right), \quad\left(s_{0} d_{2} S_{i}^{\prime}-s_{1} d_{2} S_{i}^{\prime}+S_{i}^{\prime}\right)\left(s_{1} d_{2} S_{j}^{\prime}-S_{j}^{\prime}\right)$,

which are the second order Peiffer elements defined in [5], where $S_{i} \in N E_{1}=$ $\operatorname{Ker} d_{0}=R^{+}\left[\mathbf{S}_{\mathbf{2}}\right]$ and $S_{i}^{\prime} \in N E_{2}=R\left[s_{0}\left(\mathbf{S}_{2}\right)\right]^{+}\left[s_{1}\left(\mathbf{S}_{\mathbf{2}}\right), \mathbf{S}_{\mathbf{3}}\right] \cap\left(s_{0}\left(\mathbf{S}_{\mathbf{2}}\right)-s_{1}\left(\mathbf{S}_{\mathbf{2}}\right)\right)$.

The above diagram can thus be realised as

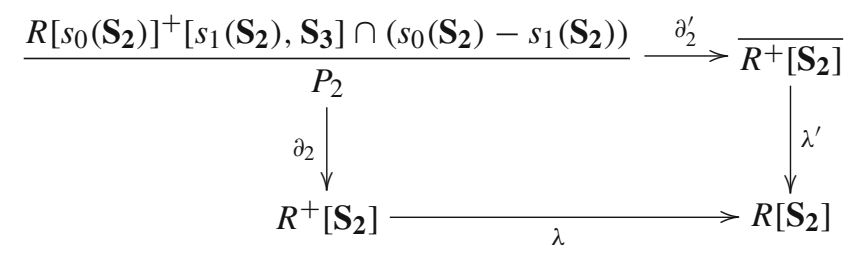

where $P_{2}$ is the second order Peiffer ideal which is in fact just $\partial_{3}\left(N E_{3}^{(2)}\right)$. 
Given any crossed square $(T, M, \bar{M}, M \rtimes R)$ with a function $v: \mathbf{S}_{\mathbf{3}} \rightarrow T$, then there exists a morphism

$$
\phi:(L, M, \bar{M}, M \rtimes R) \longrightarrow(T, M, \bar{M}, M \rtimes R)
$$

given by

$$
\phi\left(S_{i}^{\prime}+P_{2}\right)=v\left(S_{i}^{\prime}\right)
$$

such that $\phi v^{\prime}=v$ where $v^{\prime}: \mathbf{S}_{\mathbf{3}} \rightarrow L$ is a function. The existence of $\phi$ follows by using the freeness property of the algebra $N E_{2}^{(2)}$ and then restricting to $R\left[s_{0}\left(\mathbf{S}_{\mathbf{2}}\right)\right]^{+}\left[s_{1}\left(\mathbf{S}_{\mathbf{2}}\right), \mathbf{S}_{\mathbf{3}}\right] \cap\left(s_{0}\left(\mathbf{S}_{\mathbf{2}}\right)-s_{1}\left(\mathbf{S}_{\mathbf{2}}\right)\right)$. The ideal generating elements of $P_{2}$ is then easily shown to have trivial image in $T$ as that algebra is part of the second crossed square.

Thus diagram $(*)$ is the desired totally free crossed square on the 2-dimensional construction data. The crossed square properties of $(L, M, \bar{M}, M \rtimes R)$ may be easily verified or derived from the fact that this is exactly $\mathbf{M}\left(\mathbf{E}^{(2)}, 2\right)$.

Remark At this stage, it is important to note that nowhere in the argument was use made of the freeness of the 1-skeleton. If $\mathbf{E}$ is any 1-skeletal simplicial algebra and we form a new simplicial algebra $\mathbf{F}$ by adding in a set $\mathbf{S}_{3}$ of new generators in dimension 2, so that for instance, $F_{2}=E_{2}^{+}\left[\mathbf{S}_{3}\right]$, a free algebra on $\mathbf{S}_{3}$, then we can use $M=N E_{1}=\operatorname{Ker} d_{0}^{1}$ as before even though it need not be free. The corresponding $\bar{M}$ is then isomorphic to $\operatorname{Ker} d_{1}^{1}$ with the bottom right hand corner being $E_{1}$. The 'construction data' is now replaced by data for killing some elements of $\pi_{1}(E)$, specified by $f_{3}: \mathbf{S}_{3} \rightarrow M$. We introduce the term 'totally free crossed square' for the type of free crossed square constructed in the above theorem, using free crossed square for the more general situation in which $(M, E, \partial)$ and $f_{3}$ are specified and no requirement $(M, E, \partial)$ to be a free pre-crossed module is made.

\subsection{The $n$-type of the $k$-skeleton}

As in the other paper in this series, we will use the 'step-by-step' construction of a free simplicial algebra to observe the way in which the models react to the various steps of the construction.

By a 'step-by-step' construction of a free simplicial algebra, there are simplicial inclusions

$$
\mathbf{E}^{(0)} \subseteq \mathbf{E}^{(1)} \subseteq \mathbf{E}^{(2)} \subseteq \cdots
$$

The functor, $\mathbf{M}(, n)$, from the category of simplicial algebras to that of crossed $n$ cubes gives the corresponding inclusions

$$
\mathbf{M}\left(\mathbf{E}^{(0)}, n\right) \hookrightarrow \mathbf{M}\left(\mathbf{E}^{(1)}, n\right) \hookrightarrow \mathbf{M}\left(\mathbf{E}^{(2)}, n\right) \hookrightarrow \cdots
$$

We investigate $\mathbf{M}\left(\mathbf{E}^{(\mathbf{i})}, n\right)$, for $n=0,1,2$, and varying $i$. 
Firstly look at $\mathbf{M}\left(\mathbf{E}^{(\mathbf{0})}, n\right)$, where the 0-skeleton $\mathbf{E}^{(0)}$ is

$$
\mathbf{E}^{(0)}: \cdots \longrightarrow R \longrightarrow R \longrightarrow R \stackrel{f}{\longrightarrow} B
$$

with the $d_{i}^{n}=s_{j}^{n}=$ identity homomorphisms.

For $n=0$, there is an equality $\mathbf{M}\left(\mathbf{E}^{(\mathbf{0})}, 0\right)=E_{0}^{(0)} / d_{1}\left(\operatorname{Ker} d_{0}\right)=R$, and so $\mathbf{M}\left(\mathbf{E}^{(\mathbf{0})}, 0\right)$ is just an algebra of 0-simplices of $\mathbf{E}$.

For $n=1, \mathbf{M}\left(\mathbf{E}^{(\mathbf{0})}, 1\right)$ is $N E_{1}^{(0)} / \partial_{2} N E_{2}^{(0)} \rightarrow E_{0}$. It is easy to show that $N E_{1}^{(0)} / \partial_{2} N E_{2}^{(0)}$ is trivial in the 0-skeleton $\mathbf{E}^{(0)}$ and hence

$$
\mathbf{M}\left(\mathbf{E}^{(\mathbf{0})}, 1\right) \cong(0 \longrightarrow R) .
$$

For $n=2, \mathbf{M}\left(\mathbf{E}^{(\mathbf{0})}, 2\right)$ is the trivial crossed square

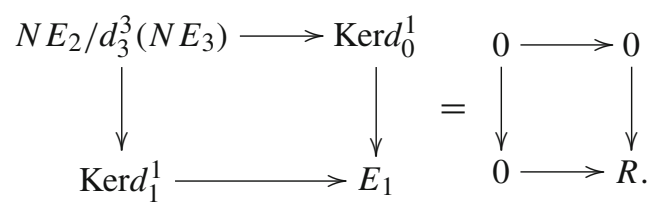

Next take $\mathbf{M}\left(\mathbf{E}^{(\mathbf{1})}, n\right)$ and recall that the 1-skeleton $\mathbf{E}^{(1)}$ is

$$
\mathbf{E}^{(\mathbf{1})}: \cdots R\left[s_{0}\left(\mathbf{S}_{2}\right), s_{1}\left(\mathbf{S}_{2}\right)\right] \underset{s_{0}, s_{1}}{\stackrel{d_{0}, d_{1}, d_{2}}{\rightleftarrows}} R\left[\mathbf{S}_{\mathbf{2}}\right] \stackrel{d_{0}, d_{1}}{\underset{s_{0}}{\rightleftarrows}} R \stackrel{f}{\rightleftarrows} R / I .
$$

For $n=0$, it follows that $\mathbf{M}\left(\mathbf{E}^{(\mathbf{1})}, 0\right)$ is $E_{0}^{(1)} / d_{1}\left(\operatorname{Ker} d_{0}\right) \cong R / I$ which is $\pi_{0}\left(\mathbf{E}^{(1)}\right) \cong$ $\pi_{0}(\mathbf{E})$.

Let $n=1$. We have that

$$
\begin{aligned}
\mathbf{M}\left(\mathbf{E}^{(1)}, 1\right) & =N E_{1}^{(1)} / \partial_{2} N E_{2}^{(1)} \rightarrow E_{0}^{(1)} \\
& =R^{+}\left[\mathbf{S}_{2}\right] / P_{1} \rightarrow R
\end{aligned}
$$

which is the free crossed module. In fact this is the free crossed module on the (generalised) presentation $\left(\mathbf{S}_{\mathbf{1}} ; \mathbf{S}_{\mathbf{2}}, f_{2}\right)$. As pointed out in [5], it is often convenient to generalise the notion of a presentation $P=\left(R ; x_{1}, \ldots, x_{n}\right)$ of an $R$-algebra $B$ in this way and $\mathbf{E}^{(1)}$ is the 1-skeleton of the free simplicial algebra generated by this presentation, then

$$
\delta: N E_{1}^{(1)} / \partial_{2}\left(N E_{2}^{(1)}\right) \longrightarrow N E_{0}^{(1)}
$$

is the free crossed module on $\left\{S_{1}, \ldots, S_{n}\right\} \rightarrow R$. This has a neat description (cf. [17]) as follows: The Peiffer ideal $P_{1}=\partial_{2}\left(N E_{2}^{(1)}\right)$ contains all such terms as $S_{i} S_{j}-\delta\left(S_{i}\right) S_{j}$, 
so any polynomial in the $S_{i}$ 's can be reduced $\bmod P_{1}$ to a linear form, hence each coset has a representative of the form $\sum r_{i} S_{i}$. As $S_{i} S_{j}=S_{j} S_{i}$, these representatives are nonunique and so the free crossed $C$ is $R^{n}$ factored by all $\delta\left(S_{i}\right) S_{j}-\delta\left(S_{j}\right) S_{i}$ we thus have

$$
\pi_{1}\left(\mathbf{M}\left(\mathbf{E}^{(1)}, 1\right)\right) \cong \operatorname{Ker}(C \longrightarrow R) \cong H_{2}(B, B)
$$

the second André-Quillen homology group, where $C \cong R^{n} / \operatorname{Im} d$, for $d: \Lambda^{2} R^{n} \rightarrow R$, the second Koszul differential, see [17] for details. Thus

$$
\begin{aligned}
& \pi_{0}\left(\mathbf{M}\left(\mathbf{E}^{(1)}, 1\right)\right) \cong B \\
& \pi_{1}\left(\mathbf{M}\left(\mathbf{E}^{(1)}, 1\right)\right) \cong H_{2}(B, B)
\end{aligned}
$$

whilst

$$
\pi_{i}\left(\mathbf{M}\left(\mathbf{E}^{(1)}, 1\right)\right) \cong 0
$$

For $n=2$,

$$
N E_{2}^{(1)}=\left(R\left[s_{0}\left(\mathbf{S}_{2}\right)\right]^{+}\left[s_{1}\left(\mathbf{S}_{\mathbf{2}}\right)\right]\right) \cap\left(s_{0}\left(\mathbf{S}_{\mathbf{2}}\right)-s_{1}\left(\mathbf{S}_{\mathbf{2}}\right)\right),
$$

$\mathbf{M}\left(\mathbf{E}^{(1)}, 2\right)$ simplifies to give (up to isomorphism)

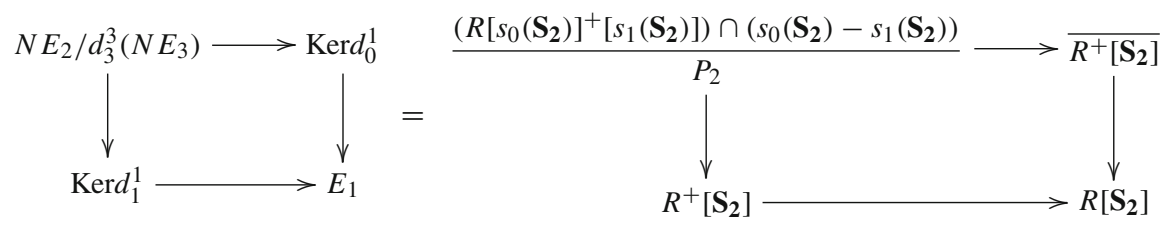

which is a crossed square.

Let us next look at $\mathbf{M}\left(\mathbf{E}^{(2)}, n\right)$. Recall the 2-skeleton $\mathbf{E}^{(2)}$

$$
\left(R\left[s_{0}\left(\mathbf{S}_{2}\right), s_{1} \mathbf{S}_{2}\right]\right)\left[\mathbf{S}_{\mathbf{3}}\right] \underset{s_{0}, s_{1}}{\stackrel{d_{0}, d_{1}, d_{2}}{\rightleftarrows}} \stackrel{\stackrel{\rightleftarrows}{\rightleftarrows}}{\rightleftarrows}\left[\mathbf{S}_{2}\right] \underset{s_{0}}{\stackrel{d_{0}, d_{1}}{\rightleftarrows}} R \stackrel{f}{\rightleftarrows} R / I .
$$

The following equalities can be easily obtained by direct calculation: for $n=0$,

$$
\mathbf{M}\left(\mathbf{E}^{(2)}, 0\right)=E_{0} / d_{1}\left(\operatorname{Ker} d_{0}\right) \cong \pi_{0}\left(\mathbf{E}^{(2)}\right)=\mathbf{M}\left(\mathbf{E}^{(1)}, 0\right) .
$$

For $n=1$,

$$
\mathbf{M}\left(\mathbf{E}^{(2)}, 1\right) \cong\left(R^{+}\left[\mathbf{S}_{2}\right] / P_{1} \rightarrow R\right)=\mathbf{M}\left(\mathbf{E}^{(1)}, 1\right)
$$


and there is an isomorphism

$$
\pi_{2}\left(\mathbf{E}^{(2)}\right) \cong \operatorname{Ker}\left(N E_{2}^{(2)} / \partial_{3}\left(N E_{3}^{(2)}\right) \longrightarrow E_{1}^{(2)}\right)
$$

Finally, let $n=2$. Since by an earlier result of this section, $\mathbf{M}\left(\mathbf{E}^{(2)}, 2\right)$ corresponds to the free crossed square, we obtain:

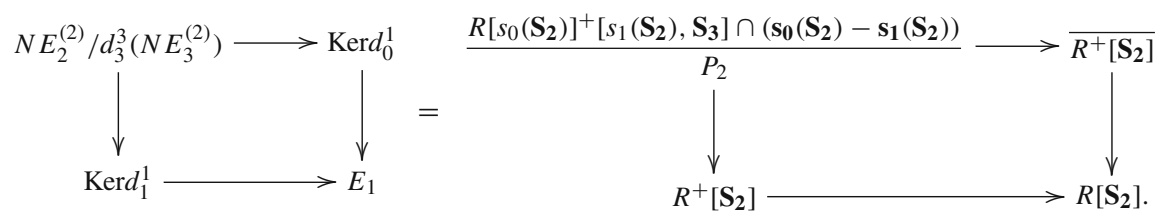

This reduces to the earlier case if $\mathbf{S}_{\mathbf{3}}$ is empty. Thus we have the following relations

$$
\mathbf{M}\left(\mathbf{E}^{(2)}, 0\right)=\mathbf{M}\left(\mathbf{E}^{(1)}, 0\right), \quad \mathbf{M}\left(\mathbf{E}^{(2)}, 1\right)=\mathbf{M}\left(\mathbf{E}^{(1)}, 1\right)
$$

but $\mathbf{M}\left(\mathbf{E}^{(2)}, 2\right)$ and $\mathbf{M}\left(\mathbf{E}^{(3)}, 2\right)$ need not to be the same due to the additional influence of $\mathbf{S}_{\mathbf{3}}$. Of course it is clear that, in general:

$$
\mathbf{M}\left(\mathbf{E}^{(i)}, n\right)=\mathbf{M}\left(\mathbf{E}^{(i+1)}, n\right) \quad \text { if } i \geq n+1 .
$$

Clearly these top left hand corner terms are unwieldy to handle and we will seek in Sect. 6 an alternative description.

\section{Squared complexes}

The first author and Koçak defined $n$-crossed complexes of algebras in [4] as the analogue for commutative algebras of the notion introduced by Ellis [12] in homotopy theory. In this paper we will only need the case $n=2$, which we shall call a squared complex; it consists of a diagram of algebra homomorphisms

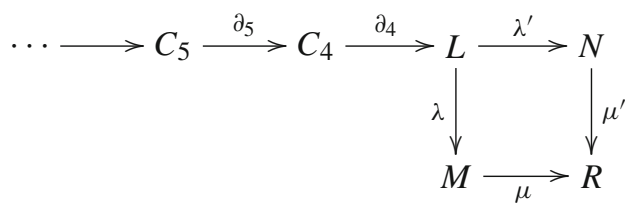

together with an action of $R$ on $L, N, M$ and $C_{i}$ for $i \geq 4$, and a function $h: M \times N \rightarrow$ $L$. The following axioms need to be satisfied. 
i. The square

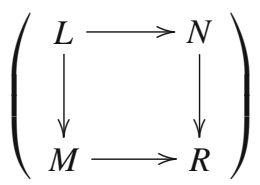

is a crossed square.

ii. $C_{n}$ is an $A$-module for $n \geq 4$ with $A=R /\left\{\mu(M)+\mu^{\prime}(N)\right\}$.

iii. The action of $R$ on $C_{n}, n \geq 4$, is such that $\mu(M)$ and $\mu^{\prime}(N)$ operate trivially. Thus each $C_{n}$ is an $A$-module.

iv. Each $\partial_{n}$ is an $A$-module homomorphism and for $n \geq 4, \partial_{n} \partial_{n+1}=0$.

A morphism of squared complexes

$$
\phi:\left(C_{i},\left(\begin{array}{ll}
L & N \\
M & R
\end{array}\right)\right) \longrightarrow\left(C_{i}^{\prime},\left(\begin{array}{ll}
L^{\prime} & N^{\prime} \\
M^{\prime} & R^{\prime}
\end{array}\right)\right)
$$

consists of a morphism of crossed squares $\left(\phi_{L}, \phi_{M}, \phi_{N}, \phi_{R}\right)$ together with a family of $\phi_{R}$-equivariant homomorphisms $\phi_{i}, i \geq 4$ satisfying $\phi_{L} \partial_{4}=\partial_{4}^{\prime} \phi_{4}$ and $\phi_{i-1} \partial_{i}=\partial_{i}^{\prime} \phi_{i}$ for $i \geq 5$. There is clearly a category SqComp of squared complexes.

By a (totally) free squared complex, we will mean one in which the crossed square is (totally) free, and in which each $C_{n}$ is free as a $\pi_{0}$-module for $i \geq 3$.

Proposition 5.1 There is a functor

$$
C(, 2): \text { SimpAlg } \longrightarrow \text { SqComp }
$$

such that free simplicial algebras are sent to totally free squared complexes.

Proof Let $\mathbf{E}$ be a simplicial algebra. We will define a squared complex $C(\mathbf{E}, 2)$ by specifying $C(\mathbf{E}, 2)_{A}$ for each $A \subseteq\langle 2\rangle$ and for $n \geq 3, C(\mathbf{E}, 2)_{n}$. As usual, (cf. the other paper in this series [5-7]), we will denote by $D_{n}$ the ideal of $N E_{n}$ generated by degenerate elements.

For $A \subseteq\langle 2\rangle$, we define in particular

$$
C(\mathbf{E}, 2)_{\langle 2\rangle}=\mathbf{M}\left(\mathbf{s k}_{2} \mathbf{E}, 2\right)_{\langle 2\rangle}=\frac{N E_{2}}{\partial_{3}\left(N E_{3} \cap D_{3}\right)} .
$$

We do not need to define $\mu_{i}$ and the $h$-maps relative to these algebras as they are already defined in the crossed square $\mathbf{M}\left(\mathbf{s k}_{2} \mathbf{E}, 2\right)_{A}$.

For $n \geq 3$, we set

$$
C(\mathbf{E}, 2)_{n}=\frac{N E_{n}}{\left(N E_{n} \cap D_{n}\right)+d_{n+1}\left(N E_{n+1} \cap D_{n+1}\right)} .
$$

As this is part of the crossed complex associated to $\mathbf{E}$, we can take the structure maps to be those of that crossed complex, cf. [5]. The terms are all modules over the 
corresponding, $\pi_{0}$ as is easily checked. The final missing piece, $\partial_{3}$, of the structure is induced by the differential $\partial_{3}$ of $N E$.

The axioms for a squared complex can now be verified using the known results for crossed squares and for crossed complexes with a direct verification of those axioms relating to the interaction of the two parts of the structure, much as in [5].

Now suppose the simplicial algebra is free. The proof of the freeness of $\mathbf{M}\left(s k_{2} \mathbf{E}, 2\right)$ together with the freeness of the crossed complex of a free simplicial algebra, [5], now completes the proof.

Suppose that $\rho$ is a general squared complex. The homotopy modules $\pi_{n}(\rho), n \geq 0$ of $\rho$ are defined in [4] to be the homology modules of the complex

$$
\cdots \stackrel{\partial_{6}}{\longrightarrow} C_{5} \stackrel{\partial_{5}}{\longrightarrow} C_{4} \stackrel{\partial_{4}}{\longrightarrow} L \stackrel{\partial_{3}}{\longrightarrow} M \rtimes N \stackrel{\partial_{2}}{\longrightarrow} R \stackrel{\partial_{1}}{\longrightarrow} 0
$$

with $\partial_{3}(l)=\left(-\lambda l, \lambda^{\prime} l\right)$ and $\partial_{2}(m, n)=\mu(m)+\mu^{\prime}(n)$. The axioms of a crossed square guarantee (see [7]) that $\partial_{3}$ and $\partial_{2}$ are homomorphisms with $\partial_{4}\left(C_{4}\right)$ an ideal in $\operatorname{Ker}\left(\partial_{3}\right), \partial_{3}(L)$ an ideal in $\operatorname{Ker}\left(\partial_{2}\right)$, and $\partial_{2}(M \rtimes N)$ an ideal in $R$.

Proposition 5.2 The homotopy groups of $C(\mathbf{E}, 2)$ are isomorphic to those of $\mathbf{E}$ itself.

Proof Again this is a consequence of well-known results on the two parts of the structure.

\section{Alternative description of freeness}

In the context of CW-complexes, Ellis [12] gave a neat description of the top algebra $L$ in (totally) free crossed squares. A free simplicial algebra is the algebraic analogue of a CW-complex so one would expect a similar result to hold in this setting. For this we need two constructions.

\subsection{Tensor products}

Suppose that $\mu: M \rightarrow R$ and $v: N \rightarrow R$ are crossed modules of commutative algebras over $R$. The algebras $M$ and $N$ act on each other, and themselves, via the action of $R$. The tensor product $M \otimes N$ is the algebra generated by the symbols $m \otimes n$ for $m \in M, n \in N$ and $r \in R$ subject to the relations

$$
\begin{aligned}
& \text { i. } r(m \otimes n)=r m \otimes n=m \otimes r n \\
& \text { ii. }\left(m+m^{\prime}\right) \otimes n=m \otimes n+m^{\prime} \otimes n \\
& \quad m \otimes\left(n+n^{\prime}\right)=m \otimes n+m \otimes n^{\prime} \\
& \text { iii. }(m \otimes n)\left(m^{\prime} \otimes n^{\prime}\right)=\left(m m^{\prime} \otimes n n^{\prime}\right)
\end{aligned}
$$

where $m^{\prime} \in M$ and $n^{\prime} \in N$. There are morphisms $\lambda: M \otimes N \rightarrow M, m \otimes n \mapsto m \cdot n=$ $m \nu(n)$ and $\lambda^{\prime}: M \otimes N \rightarrow N, m \otimes n \mapsto n \cdot m=\mu(m) n$. The algebra $R$ acts on $M \otimes N$ 
by $r \cdot(m \otimes n)=r \cdot m \otimes n=m \otimes r \cdot n$, and there is a function $h: M \times N \rightarrow M \otimes N$, $(m, n) \mapsto m \otimes n$. It is verified in [4] that this structure is a crossed square

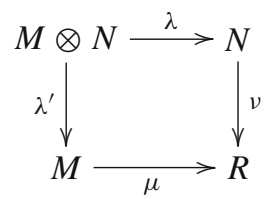

with the universal property of extending the corner

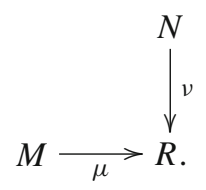

\subsection{Coproducts}

The following construction is due to Shammu [19].

Let $\left(M, R, \partial_{1}\right),\left(N, R, \partial_{2}\right)$ be crossed $R$-modules. Then $N$ acts on $M$, and $M$ acts on $N$, via the given actions of $R$. Let $M \rtimes N$ denote the semidirect product with the multiplication given by

$$
(m, n)\left(m^{\prime}, n^{\prime}\right)=\left(m m^{\prime}, n \partial_{1}\left(m^{\prime}\right)+\partial_{1}(m) n^{\prime}+n n^{\prime}\right)
$$

and injections

$$
\begin{aligned}
& i^{\prime}: M \rightarrow M \rtimes N \text { and } j^{\prime}: N \rightarrow M \rtimes N \\
& m \mapsto(m, 0) \quad n \mapsto(0, n) .
\end{aligned}
$$

We define the pre-crossed module

$$
\begin{aligned}
\underline{\delta}: & M \rtimes N \longrightarrow R \\
& (m, n) \longmapsto \partial_{1}(m)+\partial_{2}(n) .
\end{aligned}
$$

Let $P$ be the ideal of $M \rtimes N$ generated by elements of the form

$$
(m, n)\left(m^{\prime} n^{\prime}\right)-\underline{\delta}(m, n)\left(m^{\prime}, n^{\prime}\right)=\left(-\partial_{1}(m) n, m \partial_{2}(n)\right)
$$

for all $(m, n),\left(m^{\prime}, n^{\prime}\right) \in M \rtimes N$. Thus we are able to form the quotient algebra $M \rtimes N / P$ and obtain an induced morphism

$$
\partial: M \rtimes N / P \longrightarrow R
$$


given by

$$
\partial(m, n)+P=\partial_{1} m+\partial_{2} n .
$$

Let $q: M \rtimes N \rightarrow M \rtimes N / P$ be projection and let $i=q i^{\prime}, j=q j^{\prime}$. Then $M \sqcup N=$ $M \rtimes N / P$, with, the morphism $i, j$, is the coproduct of $M, N$ in the category $\mathbf{X M o d}_{R}$. The above notation can be summarised in the following diagram:

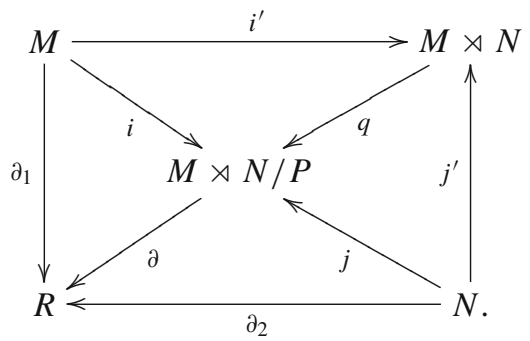

Proposition 6.1 Let

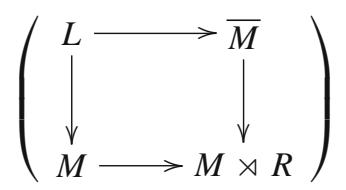

be a free crossed square on the 2-dimensional construction data or on functions $\left(f_{2}, f_{3}\right)$ as described above. Let $\partial: C \rightarrow M \rtimes R$ be the free crossed module on the function $S_{3} \rightarrow M \rtimes R$ given by $y \mapsto\left(f_{3} y, 0\right)$. Form the crossed module $\partial^{\prime}$ : $M \otimes \bar{M} \rightarrow M \rtimes R$, then

$$
L \cong\{(M \otimes \bar{M}) \sqcup C\} / \sim
$$

where $\sim$ corresponds to the relations

$$
\begin{aligned}
& \text { 1. } i_{M \otimes \bar{M}}(\partial c \otimes \bar{n}) \sim j(c)-j(\bar{n} \cdot c) \\
& \text { 2. } i_{M \otimes \bar{M}}(m \otimes \partial c) \sim j(m \cdot c)-j(c)
\end{aligned}
$$

for $c \in C, m \in M$ and $\bar{n} \in \bar{M}$.

The homomorphisms $L \rightarrow M, L \rightarrow \bar{M}$ are given by the homomorphisms

$$
\lambda: M \otimes \bar{M} \rightarrow M \quad \text { and } \quad \lambda^{\prime}: M \otimes \bar{M} \rightarrow \bar{M}
$$

and $\partial: C \rightarrow M \cap \bar{M}$. The h-map of the crossed square is given by

$$
h(m, \bar{n})=i(m \otimes \bar{n})
$$

for $m \in M, \bar{n} \in \bar{M}$. 
Proof This comes by direct verification using the universal properties of tensors and coproducts.

Remark For future applications it is again important to note that the result is not dependent on the crossed square being totally free. If $M \rightarrow R$ is any pre-crossed module, one can form the corner

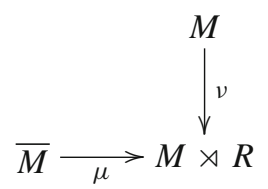

complete it to a crossed square via $M \otimes \bar{M}$ and then add in $\mathbf{S}_{\mathbf{3}} \rightarrow M$. Nowhere does this use freeness of $M \rightarrow R$.

Corollary 6.2 Let $\boldsymbol{E}^{(1)}$ be the 1-skeleton of a free simplicial algebra. Given the free crossed square $\mathbf{M}\left(\mathbf{E}^{(1)}, 2\right)$ described above, then

$$
N E_{2}^{(1)} / \partial_{3} N E_{3}^{(1)} \cong \operatorname{Kerd}_{1}^{1} \otimes_{E_{1}} \operatorname{Kerd}_{0}^{1}
$$

Proof In the 1-skeleton of a free simplicial algebra $\mathbf{E}^{(1)}$, the set $\mathbf{S}_{3}$ is empty. Thus this is clear from the previous proposition.

Remark If we set $M=\operatorname{ker} d_{0}^{1}=N E_{1}^{(1)}$, then the identification given by the Corollary gives

$$
N E_{2}^{(1)} / \partial_{3}\left(N E_{3}^{(1)}\right) \cong M \otimes \bar{M}
$$

This uses the fact that $\operatorname{ker} d_{0}^{1}$ and $\operatorname{ker} d_{1}^{1}$ are linked via the map sending $m$ to $\left(m-s_{0} d_{1} m\right)$ for $m \in \operatorname{ker} d_{0}^{1}$. The $h$-map

$$
h: M \times \bar{M} \longrightarrow N E_{2}^{(1)} / \partial_{3} N E_{3}^{(1)}
$$

is given by

$$
h(x, \bar{y})=s_{1} x\left(s_{1} y-s_{0} y\right)+\partial_{3} N E_{3}^{(1)} .
$$

But this is also $h(x, \bar{y})=x \otimes \bar{y}$. Thus

$$
x \otimes \bar{y}=s_{1} x\left(s_{1} y-s_{0} y\right)+\partial_{3} N E_{3}^{(1)}
$$

under the identification via the isomorphism of the above corollary.

This explains the mysterious formula of [6] in the discussion before Proposition 2.6 of that paper. 


\section{Applications}

\subsection{2-Crossed complexes}

A notion of 2-crossed complex of commutative algebras is defined by Grandjean and Vale in [13]. We have considered freeness conditions in [7] and this generalises easily to 2-crossed complexes.

A 2-crossed complex of commutative algebras is a sequence of $k$-algebras

$$
C: \cdots \stackrel{\partial_{n+1}}{\longrightarrow} C_{n} \stackrel{\partial_{n}}{\longrightarrow} C_{n-1} \stackrel{\partial_{n-1}}{\longrightarrow} \cdots \stackrel{\partial_{3}}{\longrightarrow} C_{2} \stackrel{\partial_{2}}{\longrightarrow} C_{1} \stackrel{\partial_{1}}{\longrightarrow} C_{0}
$$

together with a 2-crossed module structure given by the pairing

$$
\{\otimes\}: C_{1} \otimes_{C_{0}} C_{1} \longrightarrow C_{2}
$$

such that

i. $C_{n}$ is an $A$-module for $n \geq 3$ with $A=C_{0} / \partial_{1}\left(C_{1}\right)$;

ii. $C_{0}$ acts on $C_{n}, n \geq 1$, the action of $\partial_{1}\left(C_{1}\right)$ being trivial on $C_{n}$ for $n \geq 3$;

iii. Each $\partial_{n}$ is an $A$-module homomorphism and $\partial_{n} \partial_{n+1}=0$ for all $n \geq 1$.

Note that $K=\operatorname{Ker} \partial_{2}$ is an $C_{0} / \partial_{1} C_{1}$-module as $\partial_{2}$ is a crossed module.

The notion of a morphism for 2-crossed complexes should be clear. Such a morphism will be a morphism 'chain complexes of algebras' restricting to a morphism of 2 -crossed modules on the bottom three terms and compatible with the action. This gives the category, 2-CrsComp, of 2-crossed complexes and morphisms between them.

Proposition 7.1 There is a functor

$$
\mathrm{C}^{(2)}: \text { SimpAlg } \longrightarrow \text { 2-CrsComp. }
$$

(We will usually omit the superfix (2) writing simply $\mathbf{C}$ for this.)

Proof Given a simplicial algebra $\mathbf{E}$, define

$$
C_{n}= \begin{cases}N E_{n} & \text { for } n=0,1 ; \\ \frac{N E_{2}}{\partial_{3}\left(N E_{3} \cap D_{3}\right)} \quad N E_{n} & \text { for } n=2 ; \\ \frac{\left(N E_{n} \cap D_{n}\right)+\partial_{n+1}\left(N E_{n+1} \cap D_{n+1}\right)}{(N)} & \text { for } n \geq 3,\end{cases}
$$

with $\partial_{n}$ induced by the differential of NE. Note that the bottom three terms (for $n=0,1$ and 2) form the 2-crossed module considered in [7]. The Peiffer lifting map

$$
\{-\otimes-\}: N E_{1} \otimes N E_{1} \longrightarrow \frac{N E_{2}}{\partial_{3}\left(N E_{3} \cap D_{3}\right)}
$$


is given by $\{x \otimes y\}=s_{1} x\left(s_{1} y-s_{0} y\right)+\partial_{3}\left(N E_{3} \cap D_{3}\right)$ for $x, y \in N E_{1}$. The action of $a \in N E_{0}$ on $x \in N E_{n}$ for $n \geqslant 1$ is given by $a \cdot x=s_{n} s_{n-1} \ldots s_{0}(a) x$. For $n \geq 3$ the algebras are all $C_{0} / \partial_{1}\left(C_{1}\right)$-modules, since in these dimensions $C_{n}$ is the same as the corresponding crossed complex term (cf. [5]). The only thing remaining is to check that $\partial_{2} \partial_{3}$ is trivial which is straightforward.

Since

$$
\mathbf{C}^{(2)}(\mathbf{E})_{2}=N E_{2} / \partial_{3}\left(N E_{3} \cap D_{3}\right)
$$

the same formula as that for $\mathbf{C}(\mathbf{E}, 2)_{\langle 2\rangle}$, we obtain the following result.

Corollary 7.2 If $\mathbf{E}^{(1)}$ is the 1-skeleton of a free simplicial algebra $\mathbf{E}$ then the 2-crossed complex of $\mathbf{E}^{(1)}$ satisfies

$$
\mathbf{C}^{(2)}\left(\mathbf{E}^{(1)}\right)_{2} \cong \operatorname{Kerd}_{1}^{1} \otimes \operatorname{Kerd}_{0}^{1}
$$

Moreover

$$
\mathbf{C}^{(2)}\left(\mathbf{E}^{(2)}\right)_{2} \cong\left(\left(\operatorname{Kerd}_{1}^{1} \otimes \operatorname{Kerd}_{0}^{1}\right) \sqcup C\right) / \sim
$$

where as in Proposition 6.2, this $C$ is a free crossed module on the "new generators" in dimension 2.

Lemma 7.3 If $\mathbf{E}$ is a simplicial resolution of $B$ then, for $k \geq 3, \mathbf{C}^{(2)}\left(\mathbf{E}^{(2)}\right)_{k}$ a free $B$-module on the given data.

To sum up we have the following result.

Theorem 7.4 The 'step-by-step' construction of a simplicial resolution of an algebra $B$ gives a 'step-by-step' construction of a 2-crossed resolution of B via the 2-crossed complex construction $\mathbf{C}^{(2)}$.

7.2 'Quadratic' analogues of the cotangent complex?

In this final section we take the 1-skeleton of a simplicial algebra and see how it relates to other algebraic construction, such as André-Quillen homology and a squared complex form of the cotangent complex. Here our results are less conclusive than we would like.

Remark We will assume that rings and algebras are Noetherian for convenience and thus that ideals are finitely generated. This means $\mathbf{S}_{\mathbf{2}}, \mathbf{S}_{\mathbf{3}}$ etc will all be finite. 
From Sect. 4.2 we have the free crossed square

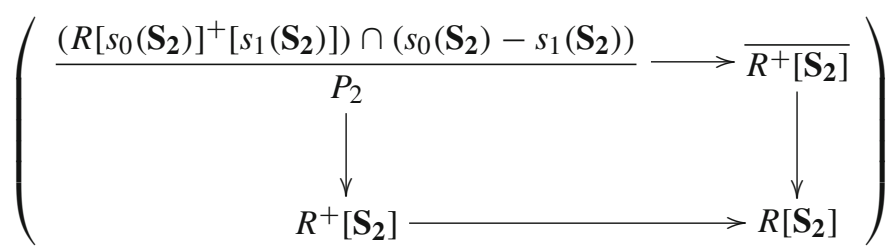

so using Corollary 6.2 there is the following isomorphism:

$$
\frac{\left(R\left[s_{0}\left(\mathbf{S}_{\mathbf{2}}\right)\right]^{+}\left[s_{1}\left(\mathbf{S}_{\mathbf{2}}\right)\right]\right) \cap\left(s_{0}\left(\mathbf{S}_{\mathbf{2}}\right)-s_{1}\left(\mathbf{S}_{\mathbf{2}}\right)\right)}{P_{2}} \cong R^{+}\left[\mathbf{S}_{\mathbf{2}}\right] \otimes \overline{R^{+}\left[\mathbf{S}_{\mathbf{2}}\right]}
$$

Thus the free crossed square becomes

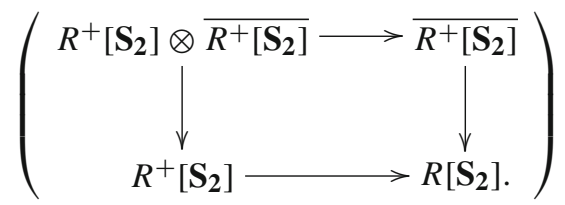

In Sect. 5 we saw that there is a 2-crossed module

$$
\mathbb{X}: \quad R^{+}\left[\mathbf{S}_{\mathbf{2}}\right] \otimes \overline{R^{+}\left[\mathbf{S}_{\mathbf{2}}\right]} \stackrel{\partial_{3}}{\longrightarrow} R^{+}\left[\mathbf{S}_{\mathbf{2}}\right] \rtimes \overline{R^{+}\left[\mathbf{S}_{\mathbf{2}}\right]} \stackrel{\partial_{2}}{\longrightarrow} R\left[\mathbf{S}_{\mathbf{2}}\right]
$$

where

$$
\partial_{3}(x \otimes y)=\left(-\lambda(x \otimes y), \lambda^{\prime}(x \otimes y)\right)
$$

and

$$
\partial_{2}(x, y)=\mu(x)+\mu^{\prime}(x) .
$$

The axioms of a squared complex ensure that $\partial_{3}$ and $\partial_{2}$ are homomorphisms and $\partial_{3}$ is a module.

The 2-crossed complex $\mathbf{C}^{(2)}\left(\mathbf{E}^{(2)}\right)$ has a smaller 2-crossed module at its base namely

$$
\mathbb{Y}: \quad R^{+}\left[\mathbf{S}_{\mathbf{2}}\right] \otimes \overline{R^{+}\left[\mathbf{S}_{\mathbf{2}}\right]} \longrightarrow R^{+}\left[\mathbf{S}_{\mathbf{2}}\right] \stackrel{\partial_{2}}{\longrightarrow} R
$$

and it is important to compare the two. In fact there is a split epimorphism $e=$ $\left(i d, e_{2}, e_{1}\right)$ from $\mathbb{X}$ to $\mathbb{Y}$ where $e_{1}: R\left[\mathbf{S}_{2}\right] \rightarrow R, e_{1}\left(S_{i}\right)=\partial\left(S_{i}\right)$, and $e_{2}: R^{+}\left[\mathbf{S}_{2}\right] \rtimes$ $\overline{R^{+}\left[\mathbf{S}_{\mathbf{2}}\right]} \rightarrow R^{+}\left[\mathbf{S}_{\mathbf{2}}\right]$ is given by $e_{2}\left(S_{i}, \overline{S_{j}}\right)=S_{i}$ and id $: R^{+}\left[\mathbf{S}_{\mathbf{2}}\right] \otimes \overline{R^{+}\left[\mathbf{S}_{\mathbf{2}}\right]} \rightarrow$ $R^{+}\left[\mathbf{S}_{2}\right] \otimes \overline{R^{+}\left[\mathbf{S}_{2}\right]}$ is identity map. This natural map is split, since there is a 2-crossed module map $i=\left(i d, i_{2}, i_{1}\right)$ from $\mathbb{Y}$ to $\mathbb{X}$ where $i_{1}$ is the inclusion map from $R$ to 
$R\left[\mathbf{S}_{2}\right]$ and $i_{2}: R^{+}\left[\mathbf{S}_{2}\right] \rightarrow R^{+}\left[\mathbf{S}_{\mathbf{2}}\right] \rtimes \overline{R^{+}\left[\mathbf{S}_{2}\right]}$ is given by $i_{2}\left(S_{i}\right)=\left(S_{i}, 0\right)$. It is easy to see that $e \circ i=i d_{\mathbb{Y}}$. The natural map $e=\left(i d, e_{1}, e_{2}\right)$ has the kernel

$$
0 \longrightarrow R^{+}\left[\mathbf{S}_{\mathbf{2}}\right] \stackrel{=}{\longrightarrow} R^{+}\left[\mathbf{S}_{2}\right]
$$

which has, of course, trivial homotopy. Thus $\mathbb{X}$ and $\mathbb{Y}$ encode the same information about the presentation of $R / I, I=\operatorname{Im} \partial_{2}$.

Crossed complexes form a category Crs which can be considered as a full subcategory of both the categories of 2-crossed complexes and of squared complexes. In the case of 2-crossed complexes, any crossed complex

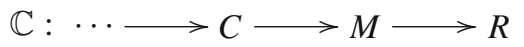

yields a 2-crossed complex with the same terms at each level and with trivial Peiffer lifting $\{\otimes\}: M \otimes_{R} M \rightarrow C$ whilst considered as a squared complex we get $\mathbb{C}$ yields

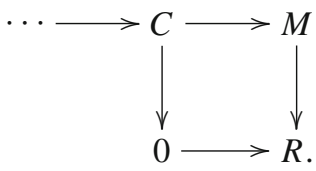

In both cases higher dimensional terms are left unchanged. Both these inclusions have left adjoints, i.e., the embeddings give reflective subcategories. The proofs are quite easily (and will be given elsewhere).

The functors from SimpAlg to 2-CrsComp and SqComp used above, when composed with the reflections to Crs yield the associated crossed complex functor mentioned earlier (see [5]).

Finally the category of chain complexes over $R / I$ embeds as a reflexive subcategory of Crs and the reflection sends a crossed resolution to the (intermediate stage of the) cotangent complex (see [5] and [18]). Thus given a simplicial resolution of $R / I$, constructed as in [1] by a step-by-step method, the 2-crossed and squared resolutions it gives can be considered as 'quadratic' analogues of the cotangent complex, in the same way that the crossed complex is a 'linear' homotopy analogue of the 'homological' cotangent complex. (Here we are using 'quadratic' and 'linear' in the analogous way to that used by Baues in [8] for the group based theory.)

Given this it is of interest to study the complex $\mathbb{X}$ (or equivalently $\mathbb{Y}$ ) and their analogues when $\mathbf{S}_{\mathbf{3}}$ information is added in. Here we have no definitive results, only problems.

The idea will be to try to provide algorithms for calculating and thus controlling, the kernel of $\partial_{3}$ in $\mathbb{X}$ (or equivalently $\mathbb{Y}$ ). We know these give $\pi_{3}\left(\mathbf{E}^{(1)}\right)$ and it is hoped that if these algorithms worked, they would allow an analysis of $\pi_{3}\left(\mathbf{E}^{(2)}\right)$ and thus to study the effect of adding in $\mathbf{S}_{\mathbf{3}}$ information to the higher terms of the simplicial resolution. As yet we are not sure if a general analysis will be possible or whether it will be necessary to limit ourselves to specific classes of example, using, for instance, methods from Gröbner base theory. 
Acknowledgments The authors wishes to thank Professor Timothy Porter and the referee for their helpful comments.

\section{References}

1. André M.: Homologie des algèbres commutatives, Die Grundlehren der Mathematischen Wissenchaften, vol. 206. Springer-Verlag, New York (1974)

2. Arvasi, Z.: Crossed squares and 2-crossed modules of commutative algebras. Theory Appl. Categories 3(7), 160-181 (1997)

3. Arvasi, Z., Ege, U.: Annihilators, multipliers and crossed modules. Appl. Categorical Struct. 11, 6 (2003)

4. Arvasi, Z., Koçak, M.: Crossed n-cubes and n-crossed complexes of commutative algebras. Turkish J. Math. 22, 127-143 (1998)

5. Arvasi, Z., Porter, T.: Simplicial and crossed resolution of commutative algebras. J. Algebra 181, 426-448 (1996)

6. Arvasi, Z., Porter, T.: Higher dimensional Peiffer elements in simplicial commutative algebras. Theory Appl. Categories 3(1), 1-23 (1997)

7. Arvas, Z., Porter, T.: Freeness conditions for 2-crossed module of commutative algebras. Appl. Categorical Struct. 6, 455-477 (1998)

8. Baues, H.J.: Combinatorial homotopy and 4-dimenional complexes, Walter de Gruyter 15, 380 (1991)

9. Duskin J.: Simplicials methods and the interpretation of triple cohomology. Memoirs AMS 3, 163 (1975)

10. Ellis, G.J.: Crossed modules and their higher dimensional analogues. Ph.D. Thesis. University of Wales, Wales (1984)

11. Ellis, G.J.: Higher dimensional crossed modules of algebras. J. Pure Appl. Algebra 52, 277-282 (1988)

12. Ellis, G.J.: Crossed squares and combinatorial homotopy. Math. Z. 214, 93-110 (1993)

13. Grandjeán, A.R., Vale, M.J.: 2-modulos cruzados an la cohomologia de André-Quillen. Memorias de la Real Academia de Ciencias 22, 1-28 (1986) (see also Linear methods in André-Quillen cohomology. C. R. Math. Rep. Acad. Sci. Canada 10, 243-248 (1988))

14. Guin-Walery, D., Loday J.-L.: Obstructions a l'excision en K-theorie algébrique, Evanston conference on algebraic K-theory, 1980. In: Lecture Notes in Mathematics, vol. 854. Springer-Verlag, New York, pp 179-216 (1981)

15. Illusie, L.: Complex cotangent et deformations I, II. In: Lecture Notes in Mathematics, vol. 239. Springer, New York (1971) (II: 283 (1972))

16. Mutlu, A., Porter, T.: Freeness conditions for crossed squares and squared complexes. K Theory 20, 345-368 (2000)

17. Porter, T.: Homology of commutative algebras and an invariant of Simis and Vasconceles. J. Algebra 99, 458-465 (1986)

18. Porter, T.: Some categorical results in the theory of crossed modules in commutative algebras. J. Algebra 109, 415-429 (1987)

19. Shammu, N.M.: Algebraic and categorical structure of category of crossed modules of algebras. Ph.D. Thesis. University of Wales, Wales (1992)

20. Whitehead, J.H.C.: Combinatorial homotopy II. Bull. Am. Math. Soc. 55, 453-496 (1949) 